\title{
A Probe into Test Design of Reading Comprehension in College English
}

\author{
Yingfei Qu \\ Foreign language School, Huaqiao University, Xiamen Campus, No. 668, Jimei Avenue, Xiamen, \\ Fujian Province, 361021, China \\ quyingfe@hqu.edu.cn,36097865@qq.com
}

Keywords: Test design; Reading comprehension; College English

\begin{abstract}
Evaluation is one of the indispensable steps in foreign language teaching. Traditionally, people in China focused too much on the scores and ignored to a large extent the gradual development. Reading is an important part in college English in China and should be evaluated scientifically. This paper, based on the theories of language testing, discussed the testing range of reading in college English.
\end{abstract}

\section{Introduction}

In most countries around the world, educational testing is increasingly administrated on a large scale. In heavily populated countries a single test may be administrated to millions of students in a single year, this is especially true for China. Generally, a large-scale testing serves three major functions: 1) selecting and placing students for further educational and career opportunities; 2) certifying that students posses requisite knowledge and skills, and 3) monitoring the educations of educational system to insure that it is functioning effectively. In order to construct tests that carry out system-oriented functions with large numbers of students, test makers have traditionally been forced to rely on multiple-choice questions. Defenders of multiple-choice tests often point to a further advantage such as money saving, objective and easy manipulation. Within such a framework, the users of the test only focus on the final stage of scoring and thus ignore the fact that language learning achievement is built in a step by step manner. In some cases, the competence even can not be reflected in the so called objective testing. Reading, as one of the most important part in college English in China, has always been a hot topic in testing. This paper probes into the merits and demerits of traditional English testing in China, and provides some suggestions in the range and scope of reading in college English testing.

\section{Relative Theories about Language Assessment}

Basic Testing Theories. In resent years, researchers on educational testing and assessment have become increasingly aware that multiple-choice questions can set up a misleading opposition between different ways of reading a text. The test is given at two levels: first level, a basic qualification, and executive level, and advanced qualification.[1] when test maker design questions, they should pay attention on it. Although not much information is available in the specimen material on the scope of the investigation into the target language use situation, it would appear that some consultations with experienced business professional were carried out. In fact, the lack of information about precisely what the criteria for correctness are is a rather serious deficiency in the specimen material.

In specific purpose language testing, we must be careful to ensure that the intended discourse domain, that of the target language use situation, is well signaled in the test[1]. Researchers use the term discourse domain to refer to the internal interpretation of context. They define discourse domain as a cognitive construct created by a language learner as a context for interlanguage development and use. There is considerable research in language testing that demonstrates the effects of test method on test performance. The characteristics of the tasks used are always likely to affect test scores to some degree, so that there is virtually no test that yields only information about the ability we want to measure. 
Pragmatic Knowledge. Pragmatic knowledge enables us to create or interpret discourse by relating utterances or sentences and texts to their meanings, to the intentions or language users, and to relevant characteristics of language use setting. According to Dai and He [2], there are two areas of pragmatic knowledge: Pragmalinguistics and Sociopragmatics. Pragamalinguistics is applied to the more linguistic end of pragmatics, i.e. how the linguistic forms are used to serve specific pragmatic purpose. Sociopragmatics is the sociological interface of pragmatics; it is concerned with the customary ways in which people of a particular culture behave to achieve a particular purpose.

Functional Knowledge. Functional knowledge is what Bachman calls "illocutionary competence" [3]. It enables us to interpret relationship between utterances of or sentences and texts and the intentions of language users. Quite frequently the appropriate interpretation of a given utterance also involves the language users' prior knowledge of the language setting, including the characteristics of the participants, their background knowledge of the world, etc. Meanwhile, to understand the so called "illocutionary" act, one must be able to analyze meaning in the language, or in other words, becomes more analytical in dealing with the indirect meaning. This, of course is the abilities test designer are interested in during their preparation of the test.

\section{The Nature of Reading Comprehension in College English}

Researchers in native language and foreign language teaching have contributed much to the understanding of reading comprehension. Reading is to get information, but it is not a unilateral process. As many researchers argued, reading is in reality a bilateral or interactive process in which there could be certain "talking" between the readers and the author. In other words, while reading, the reader is trying to find what the author wanted to express. As it is, reading comprehension must care if the material is authentic or not, that is, if there is real interaction between the reader and the author [4]. On the other hand, considering the process of language acquisition, reading is in fact a channel to provide students input which aims to promote students' learning.

One important of the interactive feature of reading is its "schemata". That is, in reading, readers tend to use the existing schemata existing in their mind, which is useful in reading. Meanwhile, reading ability lies in the understanding of the contents. This ability cannot be observed directly as it is per se the mental manipulation. However, it can be observed indirectly through the examination of some items by testing. For college English, students are supposed to master more than 5000 thousand words and be able to read passages of about 500 or more words. All this must be taken consideration in the testing system.

\section{The Issues of Testing Reading Comprehension in College English}

Relative Matters about the Design. In spite of the wide range of reading material specially written or adapted for English language learning purposes, there are few comprehensive systematic programmers which have been constructed from a detailed analysis of the skills required for efficient reading. Much test material is still limited to short reading extracts on which general 'comprehension' questions are based. Characteristics of the test rubric include the objective, procedures for responding, structure of the test/ task, time allotment [1].

The term rubric is a somewhat obscure one, originally used in Christian prayer books to refer to procedural information about the form of worship, separate from the religious content. In testing, the term has been defined by Bachman as characteristics 'that specify how test takers are expected to proceed in taking the test, and include the instructions, time allocation, and test organization. [1] The characteristic of the rubric relate to the nature of the communicative event, whether in the target language situation or the language test, including its objective, procedures for responding, structure, format, the time available for completing it, and evaluation criteria and procedures.

According to what have been discussed, it is clear that multiple-choice testing of reading comprehension cannot satisfy the requirement of measuring the abilities of reading comprehension of Chinese college students, the author present some test design to improve this problem on the basis of some relative assessment theories. The following four items about testing reading 
comprehension are proposed.

Completion Items. Completion items measure recall rather than recognition. Although such items are similar in many ways to open-ended questions in tests of reading comprehension, they often regarded as belonging more to the objective category of test items. Usually completion items require the testees to supply a word or a short phrase. Unless great care is taken to ensure that there is only one correct answer, the making will prove very difficult when the tester is confronted with a variety of answers ranging from acceptable. All valid interpretations, whether or not these were in the test writer's mind at time of the construction of the test, must be regarded as correct. In designing reading comprehension, the item is like "The author was surprised to meet Dr Steven because __ " rather than "Why was the author surprised to meet Dr Steven?"This kind of testing is often used to relatively detailed illustration which call forth students recall rather than recognition [5]. This is especially useful in classroom evaluation as well as in formal testing.

Rearrangement Items. These two item types are particularly useful for testing the ability to understand a sequence of steps of steps in a process or events in a narrative. While in an exercise for classroom practice the students will often be required to rewrite the jumbled sentences in their correct sequence, it is obviously preferable for testing purposes to instruct them to write simply the numbers or letters of the jumbled sentences.

- Type 1: the first of these items takes the form of jumbled sentences which the students have to "unscramble" and arrange in the correct order.

- Type 2: the jumbled sentences in the second item are based on a reading comprehension text and should be unscrambled in the light of the information contained in the text. The students must write the letters of the sentences again in their correct order in the boxes at the end of the item.

Open-ended and Miscellaneous Items. The term "open-ended" is used to refer to those questions which elicit a completely subjective response on the part of the testees. The response required may range from a one-word answer to one or two sentences. When making open-ended items which require answers in sentences, it is frequently advisable to award at least two or three marks for each correct answer. When constructing a reading comprehension test, the test writer should remember to let the text itself determine the types of items set. Indeed, if this principle is observed, it follows that different parts of a particular text will frequently require different item types .It is now becoming common practice for more than one type of item to be used to test comprehension of the same text.

Short-answer Questions. First of all, think-aloud protocols will be used to elicit the response of college English students to perspective test material. Second, this reader response will be used to: a) construct different ways of reading the passage and b) develop SAQs that are appropriate for Chinese university student.

Third, the SAQs will be pilot tested to determine not only psychometric properties of reliability and validity but also the degree to which they elicit: a) correct responses that are stable in form and b) in correct response that can be lexically differentiated from the correct ones (these two conditions must be met if artificial intelligence techniques are to be used in scoring response to SAQs).

Fourth, on the basis of the pilot testing, the SAQs will be revised and administrated on a large scale. Finally, a computer-based program will be developed to score selected samples of the revised SAQs (the goal here is simply to demonstrate that such programme can be developed for scoring responses to SAQs).

\section{Conclusion}

Reading comprehension is an indispensable part in college English testing system. For years in China, the tests of various types adopted merely multiple choices due to the influence of psychometric theories of the western world. Although it is admitted that this type of items are feasible in manipulation and used for years, its disadvantages are found by many researchers. For instance, it cannot reflect the real abilities of testees. Therefore, in college English teaching framework, a useful and multi-dimensional testing system must be adopted so that students' abilities 
like skimming, scanning, understanding of the details, memory, recognition, etc can be measured objectively. This paper is theoretically based, and it is hoped that in the future, more and more teachers, researchers will be involved for the reform and practice of testing students' reading comprehension in college English.

\section{References}

[1] D., Douglas, Assessing language for specific purposes. Cambridge: Cambridge University Press, 2000.

[2] W. D., Dai, \& Z. X., He. A New Concise Course in Linguistics for Students in English. Shanghai: Shanghai Foreign Language Education Press, 2010.

[3] L., Bachman. Fundamental Consideration in Language Testing. Oxford: Oxford University Press, 1998.

[4] F. F., Zhao. An Analysis of the Authenticity of Reading Comprehension in CET-4. Journal of Hubei Correspondence University, Vol.28, 2015(23), Pp168-169.

[5] J. B., Heaton. Writing English Language Tests. Beijing: foreign Language Teaching and Research Press, 2000. 